YUMS-98/11

September, 1998

hep-ph/9903214

\title{
Stop Squark Search at Tevatron in the Light Slepton Scenario
}

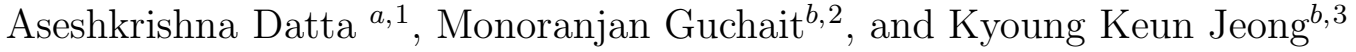 \\ ${ }^{a}$ Department of Physics, Jadavpur University, Calcutta - 700032, India \\ ${ }^{b}$ Department of Physics, Yonsei University, Seoul 120-749, Korea
}

\begin{abstract}
In minimal supergravity based supersymmetry models the charged sleptons as well as the sneutrinos may be lighter than the squarks. In such light slepton scenario the search for lighter stop squark has been investigated in the dilepton + missing $p_{T}\left(p_{T}\right)$ + jets $(\geq 1)$ channel at Tevatron. In this scenario semileptonic decay of lighter stop squark has been considered via off shell and on shell charginos. In the latter case the chargino undergoes pure leptonic 2-body decay. We observe that for some favourable region of the MSSM parameter space one can probe lighter stop squark mass upto 140 $\mathrm{GeV}$ with few events in the present data set for which the luminosity is $110 \mathrm{pb}^{-1}$. At the MI upgrade one is likely to end up with more events.
\end{abstract}

\footnotetext{
${ }^{1}$ E-Mail: asesh@juphys.ernet.in

${ }^{2}$ E-Mail: guchait@cskim.yonsei.ac.kr

${ }^{3}$ E-Mail: kkjeong@theory.yonsei.ac.kr
} 


\section{Introduction}

It is well known that supersymmetry (SUSY) [1] is a very elegant theoretical framework which basically invokes a symmetry between fermions and bosons. The Minimal Supersymmetric extension of the Standard Model(SM), popularly known as the MSSM, stands out as the most attractive model to go beyond the SM. Although it cures the SM from many of its ills, one has to pay the price in terms of a large number of free parameters and a plethora of predicted new fundamental supersymmetric particles (the SUSY particles or sparticles). On the face of the fact that the predictions of the SM have been verified very precisely at recent LEP experiments [2] and none of these sparticles has still been discovered it should be kept in mind all along that these have not contradicted any of the MSSM predictions either. Naturally search for SUSY has become a thrust area in the phenomenological studies and experiments at the present and future generation of colliders.

Based on the gauge group $S U(3)_{c} \otimes S U(2)_{L} \otimes U(1)_{Y}$ alike the SM, the MSSM contains ordinary SM particles and their corresponding superpartners which differ by spin $1 / 2$. In order to generate masses for both up and down type quarks by electroweak symmetry breaking the MSSM requires at least two $S U(2)$ higgs doublets. After electroweak symmetry breaking one is left with five physical higgses - two charged $\left(H^{ \pm}\right)$higgses, two neutral $\mathrm{CP}$ even $(h, H)$ higgses and one CP odd (pseudoscalar) higgs $(A)$ [3]. The supersymmetric partners of the charged and the neutral SM gauge bosons, called gauginos, are also not the physical states. They get mixed up because of electroweak symmetry breaking leading to physical supersymmetric states (the mass eigenstates) called charginos $\left(\tilde{W}_{i} ; i=1,2\right)$ and neutralinos $\left(\tilde{Z}_{j} ; j=1,2,3,4\right)$. The charginos and the neutralinos are the linear compositions of charged $\left(\tilde{W}^{ \pm}, \tilde{H}^{ \pm}\right)$and neutral $\left(\tilde{W}_{3}, \tilde{B}, \tilde{H}^{0}{ }_{1}, \tilde{H}^{0}{ }_{2}\right)$ gauginos respectively [1]. In this paper we shall assume $\tilde{W}_{1}$ as the lighter chargino and $\tilde{Z}_{1}$ as the lightest neutralino. In most of the models the lightest neutralino, $\tilde{Z}_{1}$ is assumed to be the lightest supersymmetric particle(LSP). In models with conserved R-parity [4] the LSP turns out to be stable and all other heavy sparticles eventually cascade to the LSP. Since the LSP must be neutral and weakly interacting on cosmological ground it leads to missing energy which is the characteristic SUSY signal. Also, the left and right handed SM fermions have their respective spin zero supersymmetric states called sfermions viz. $\tilde{f}_{L}$ and $\tilde{f}_{R}$. The mixing between the chiral sfermions are proportional to the masses of their corresponding SM partners. Therefore, such mixing becomes important only for the third generation of sfermions.

In the last few years, extensive searches for SUSY were carried out at LEP [5] as well as Tevatron [6] experiments. Non-observation of any SUSY signal excluded certain region of the MSSM parameter space and put lower bounds on different sparticle masses. However, it is to be noted that these searches were performed under some specific assumptions in the 
models and on the masses of the sparticles. In most of the cases these assumptions made the analyses very simple but were in no way compelling. Moreover, these restrict a variety of other phenomenological possibilities in the context of SUSY searches. In this work we have addressed one such issue in the context of stop squark search at Tevatron.

Due to the generic large top Yukawa coupling, the third generation of squarks viz. the stop squark, undergoes substantial mixing between the left $\left(\tilde{t}_{L}\right)$ and the right $\left(\tilde{t}_{R}\right)$ chiral states [7]. As a consequence, one of the physical states $\left(\tilde{t}_{1}\right)$ may be lighter than all other squarks in the MSSM. If this be the actual case and also that the mass of $\tilde{t}_{1}\left(m_{\tilde{t}_{1}}\right)$ is less than the gluino (the SUSY partner of the gluon) mass $\left(m_{\tilde{g}}\right)$ then $\tilde{t}_{1}$ can decay into various channels:

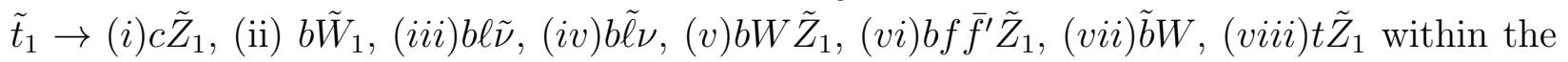
MSSM. The dominant decay channel of $\tilde{t}_{1}$ is mostly determined by $m_{\tilde{t}_{1}}$ and by the masses of the final state particles. In the case of $m_{\tilde{t}_{1}}<m_{t}, m_{\tilde{b}}$, the decay modes (vii) and (viii) are kinematically forbidden. The decay mode (vi) consists of four body final states where as the decay mode (v) involves massive particles in the final state and it is also propagator suppressed with top quark in the propagator. Therefore, whenever any one (or more) of the channels (i) - (iv), which are primarily two or three body decays, opens up that(those) will dominate over other multi-body decay processes viz,(v)-(viii) [8]. On the other hand, if the masses of the sleptons $\left(m_{\tilde{\nu}}, m_{\tilde{\ell}}\right)$ are much larger than those of $\tilde{W}_{1}$ and $\tilde{Z}_{1}$, the decay modes (iii) and (iv) are suppressed heavily in comparison to the decay modes (i) and (ii). Therefore, within this scenario where all other sparticles, except LSP, are much larger heavier than $\tilde{t} 1_{1}$, the latte has only two accessible decay modes viz. either (i) or (ii). If $m_{\tilde{t}_{1}}>m_{\tilde{W}_{1}}+m_{b}$ then the charged current decay

$$
\tilde{t}_{1} \rightarrow b \tilde{W}_{1}
$$

will be the dominant one. Otherwise it will decay via loops [8] with flavour changing neutral currents (FCNC) [8]

$$
\tilde{t}_{1} \rightarrow c \tilde{Z}_{1}
$$

and the cross section of such a process is naturally very small. In the latter case the stop pair production will lead to jets plus missing energy carried by the LSP signal[9]. In the former case ( Eq. 国), the chargino will decay via $W$ (real or virtual) as

$$
\tilde{W}_{1} \rightarrow \tilde{Z}_{1} f \bar{f}^{\prime}
$$

under the assumption that the sleptons and the squarks are heavy. Therefore, if $\tilde{W}_{1}$ decays leptonically, its decay branching ratio(BR) is essentially the BR of $W$ in the leptonic channel which is $2 / 9$ for both $e$ and $\mu$. This leptonic decay of $\tilde{W}_{1}$ leads to missing energy plus single lepton or unlike sign dileptons [9]. The decay channel of $\tilde{W}_{1}$ as given by Eq. 3 is assumed to be the only decay process of $\tilde{W}_{1}$ in most SUSY searches. Hereafter we shall call those as 
conventional SUSY searches. In theoretical studies where stop squark searches were carried out either directly [9, 10 or indirectly [11] in the context of Tevatron, the decay patterns of $\tilde{t}_{1}$ and $\tilde{W}_{1}$ are assumed to be identical as we have described above.

If the mass hierarchy among sparticles is such that the sneutrinos $(\tilde{\nu})$ [12 are much heavier than the LSP $\left(\tilde{Z}_{1}\right)$ only and lighter than all other sparticles then $\tilde{W}_{1}$ dominantly undergoes two body decay as

$$
\tilde{W}_{1} \rightarrow \ell \tilde{\nu}_{\ell}
$$

in contrast to Eq.3, with a BR $2 / 3$ where $\ell=e$ and $\mu$. Note that this two body BR of $\tilde{W}_{1}$ is larger than that for the leptonic decay mode of Eq. 3 by a factor of 3 . If the associated left handed charged slepton $\left(\tilde{\ell}_{L}\right)$ is also lighter than $\tilde{W}_{1}$ then $\tilde{W}_{1}$ can also decay in the two body channel

$$
\tilde{W}_{1} \rightarrow \tilde{\ell}_{L} \nu_{\ell}
$$

followed by

$$
\tilde{\ell}_{L} \stackrel{100 \%}{\longrightarrow} \ell \tilde{Z}_{1}
$$

In this light sneutrino scenario, the sneutrino decays in the invisible channel $\tilde{\nu} \rightarrow \nu \tilde{Z}_{1}$ with $100 \%$ BR whenever $\tilde{Z}_{1}$ has some amount of gaugino content and thus leads to missing energy in the final state. Therefore, in addition to $\tilde{Z}_{1}, \tilde{\nu}$ will behave like a LSP in such a scenario. Interestingly, it is seen that when $m_{\tilde{\nu}\left(\tilde{l}_{L}\right)}+m_{b}<m_{\tilde{t}_{1}}<m_{\tilde{W}_{1}}+m_{b}, \tilde{t}_{1}$ will decay via off-shell $\tilde{W}_{1}$ either in the channel (iii) or (iv) [8] i.e.

$$
\tilde{t}_{1} \rightarrow b \tilde{W}_{1}^{*} \rightarrow b\left(\ell \tilde{\nu}_{\ell}\right)
$$

or

$$
\tilde{t}_{1} \rightarrow b \tilde{W}_{1}^{*} \rightarrow b\left(\tilde{\ell}_{L} \nu_{\ell}\right)
$$

These three body decay modes of $\tilde{t}_{1}$ via off-shell $\tilde{W}_{1}$ always dominate over the loop level decay mode (i). Hence, most of the time the pair production of stop squark leads to unlike sign dilepton plus jets accompanied by missing energy due to the presence of $\tilde{\nu}$ and $\tilde{Z}_{1}$. In the above we have put subscript $\mathrm{L}$ in $\tilde{\ell}$ to imply that gauge dominated $\tilde{W}_{1}$ interacts with left handed sleptons only.

In Ref. [9], a study on stop squark search has been carried out in the $1 \ell+b+j e t s+\not_{T}$ and $\ell^{+} \ell^{\prime-}+$ jets $+E_{T}$ channels for the leptonic decays of $\tilde{W}_{1}$ (Eq. 3) when $m_{\tilde{t}_{1}}>m_{b}+m_{\tilde{W}_{1}}$ In this scenario appreciable jet activitys are expected as $\widetilde{W}_{1}$ decays hadronically (about $80 \%$ ). On the other hand, in the light slepton scenario (where we can have sleptons lighter than $\tilde{t}_{1}$ ) stop squark will always decay semileptonically with less jet activities as $\tilde{W}_{1}$ will not have any hadronic decay mode (Eqs. 4-8). As a consequence, the events containing only single lepton will be absent and the jets $+\not E_{T}$ signal will be degraded. Indeed, in Ref.[9] 
the authors have mentioned the possibility of enhancement of the dilepton signal for light sleptons. As we have mentioned above, this enhancement (by a factor of 10) of signal events occurs due to the eventually larger BR for the leptonic decay of $\tilde{W}_{1}$. On the other hand, in our scenario leptons are comparatively softer when $\tilde{t}_{1}$ undergoes the two body decay (Eq. 1) whereas leptons are harder in the case of its three body decay via off shell $\tilde{W}_{1}$. In view of these facts it is instructive to study the prospect of stop searches systematically for the relevent MSSM parameter space in this light slepton scenario. To the best of our knowledge, so far there is no such study at Tevatron energies, although experimental searches for $\tilde{t}_{1}$ has already been carried out at LEP in this scenario [13]. In our analysis we have taken into account the presently available experimental bounds on the relevant MSSM parameters. The phenomenological impacts of the light slepton scenario in the context of SUSY searches have been discussed in detail in a series of works [14]. This work adds to that series.

The paper is organised as follows. In section 2 we describe very briefly the stop squark masses and mixing and also present relevant formulae for the decay width of stop squark. In section 3 we discuss our results.

\section{Relevant formulae}

As already mentioned in the introduction, due to the large Yukawa interactions, the left $\left(\tilde{t}_{L}\right)$ and the right $\left(\tilde{t}_{R}\right)$ handed stop squarks get mixed up [7]. The stop mixing matrix in the basis $\left(\tilde{t}_{L}, \tilde{t}_{R}\right)$ is expressed as

$$
M_{\tilde{t}}^{2}=\left(\begin{array}{cc}
m_{\tilde{t}_{L}}^{2} & a_{t} m_{t} \\
a_{t} m_{t} & m_{\tilde{t}_{R}}^{2}
\end{array}\right)
$$

where

$$
\begin{aligned}
m_{\tilde{t}_{L}}^{2} & =m_{\tilde{Q}_{3}}^{2}+m_{t}^{2}+\left(\frac{1}{2}-\frac{2}{3} \sin ^{2} \theta_{W}\right) M_{Z}^{2} \cos 2 \beta \\
m_{\tilde{t}_{R}}^{2} & =m_{\tilde{U}_{3}}^{2}+m_{t}^{2}+\frac{2}{3} M_{Z}^{2} \sin ^{2} \theta_{W} \cos 2 \beta \\
a_{t} & =\left(A_{t}+\mu \cot \beta\right)
\end{aligned}
$$

and $\tan \beta, \mu$ and $A_{t}$ are the ratio of vacuum expectation values $\left(\tan \beta=v_{1} / v_{2}\right)$ of two higgs doublets, the SUSY higgs mass parameter and the trilinear coupling respectively. The soft mass terms for the third generation of doublet, $m_{\tilde{Q}_{3}}$ and the up type singlet, $m_{\tilde{U}_{3}}$ are related to that corresponding to the first/second generation of squarks as

$$
\begin{aligned}
& m_{\tilde{Q}_{3}}^{2}=m_{\tilde{Q}_{1}}^{2}-I \\
& m_{\tilde{U}_{3}}^{2}=m_{\tilde{U}_{1}}^{2}-I
\end{aligned}
$$


where $I$ is a function of Yukawa coupling determined by the renormalization group equation in minimal supergravity models assuming GUT scenario. The physical stop squark states $\tilde{t}_{1}$ and $\tilde{t_{2}}$ are related to the chiral states as

$$
\left(\begin{array}{c}
\tilde{t}_{1} \\
\tilde{t}_{2}
\end{array}\right)=\left(\begin{array}{cc}
\cos \theta_{\tilde{t}} & -\sin \theta_{\tilde{t}} \\
\sin \theta_{\tilde{t}} & \cos \theta_{\tilde{t}}
\end{array}\right)\left(\begin{array}{c}
\tilde{t}_{L} \\
\tilde{t}_{R}
\end{array}\right)
$$

where the mixing angle $\theta_{\tilde{t}}$ is given by

$$
\tan 2 \theta_{\tilde{t}}=\frac{2 a_{t} m_{t}}{m_{\tilde{t}_{R}}^{2}-m_{\tilde{t}_{L}}^{2}}
$$

and the mass eigenvalues are given by

$$
m_{\tilde{t}_{1,2}}^{2}=\frac{1}{2}\left[m_{\tilde{t}_{L}}^{2}+m_{\tilde{t}_{R}}^{2} \mp\left\{\left(m_{\tilde{t}_{L}}^{2}-m_{\tilde{t}_{R}}^{2}\right)^{2}+\left(2 a_{t} m_{t}\right)^{2}\right\}^{1 / 2}\right] .
$$

The diagonal terms in the stop mass matrix of Eq. 9 are very small because of Eq. 10 where they receive the large negative contribution from $I$ which depends on the large top Yukawa coupling. If we notice the expression for the mass eigenvalues (Eq. 13), it is quite obvious that if the SUSY parameters are of the same order of magnitude then $m_{\tilde{t}_{1}}$ would be very light and it may even be lighter than the top quark unlike other squark masses. Eq. 12 implies that the mixing angle is proportional to the mass of the top quark which in turn implies that such mixings for other generation of squarks are very small due to their small masses. In the following we discuss very briefly about the possible decay modes of the lighter stop $\operatorname{squark}\left(\tilde{t}_{1}\right)((\mathrm{i})-(\mathrm{iv}))$ which are relevant in our scenario.

(i) $\tilde{\mathbf{t}_{1}} \rightarrow \mathbf{c} \tilde{\mathbf{Z}}_{1}:$ It is the only allowed decay mode of $\tilde{t}_{1}$ when $m_{\tilde{t}_{1}}$ is lighter than all other sparticles except the LSP. It is shown[8] that this flavour changing decay proceeds through various loops which are logarithmically divergent. The logarithmic part of these loop diagrams induce the mixing between $\tilde{c}_{L}, \tilde{t}_{L}$ and $\tilde{t}_{R}$ whereas $\tilde{c}_{R}$ does not mix with $\tilde{t}_{L}$ and $\tilde{t}_{R}$ in the limit $m_{c} \rightarrow 0$. The corresponding decay width of $\tilde{t}_{1}$ in this flavour changing mode is

$$
\Gamma\left(\tilde{t_{1}} \rightarrow c \tilde{Z}_{1}\right)=\frac{g^{2}}{16 \pi}|\epsilon|^{2} f^{2} m_{\tilde{t}_{1}}\left(1-\frac{m_{\tilde{Z}_{1}}^{2}}{m_{\tilde{t}_{1}}^{2}}\right)^{2}
$$

where

$$
f=\sqrt{2} \frac{N_{12}}{\cos \theta_{W}}\left(\frac{1}{2}-\frac{2}{3} \sin ^{2} \theta_{W}\right)+\frac{2 \sqrt{2}}{3} N_{11} \sin \theta_{W}
$$

$N_{11}, N_{12}$ being the $\tilde{B}$ and $\tilde{W}_{3}$ components of $\tilde{Z}_{1}$ state 15. Here $\epsilon$ is the $\tilde{c}_{L}-\tilde{t}_{L}$ mixing parameter and is significantly very small $\left(\simeq 10^{-4}\right)[8]$. This leads to a moderate decay width $\Gamma\left(\tilde{t}_{1} \rightarrow c \tilde{Z}_{1}\right)$. Therefore, the other decay modes (ii)-(iv), whenever kinematically allowed, will dominate over the present mode. 
(ii) $\tilde{\mathbf{t}_{1}} \rightarrow \mathbf{b} \tilde{\mathbf{W}}_{\mathbf{1}}:$ Here the two body decay of $\tilde{t}_{1}$ proceeds through tree level charged current interaction when $m_{\tilde{t}_{1}}>m_{b}+m_{\tilde{W}_{1}}$. The decay width is

$$
\begin{aligned}
\Gamma\left(\tilde{t}_{1} \rightarrow b \tilde{W}_{1}\right) & =\frac{\alpha}{4 \sin ^{2} \theta_{W}} m_{\tilde{t}_{1}} \lambda^{1 / 2}\left(1, \frac{m_{b}^{2}}{m_{\tilde{t}_{1}}^{2}}, \frac{m_{\tilde{W}_{1}}^{2}}{m_{\tilde{t}_{1}}^{2}}\right) \\
& \times\left[\left\{\left|c_{L}\right|^{2}+\left|c_{R}\right|^{2}\right\}\left(1-\frac{m_{b}^{2}}{m_{\tilde{t}_{1}}^{2}}-\frac{m_{\tilde{W}_{1}}^{2}}{m_{\tilde{t}_{1}}^{2}}\right)-\frac{4 m_{b} m_{\tilde{W}_{1}}}{m_{\tilde{t}_{1}}^{2}} \operatorname{Re}\left(c_{L} c_{R}^{*}\right)\right] \\
c_{L} & \equiv-\frac{m_{b} U_{12}}{\sqrt{2} m_{W} \cos \beta} \cos \theta_{\tilde{t}} \\
c_{R} & \equiv V_{11} \cos \theta_{\tilde{t}}+\frac{m_{t} V_{12} \sin \theta_{\tilde{t}}}{\sqrt{2} m_{W} \sin \beta}
\end{aligned}
$$

where $U, V$ are the chargino mixing matrix [15] corresponding to the right and the left handed states respectively. Since it is a charged current two body decay process, it will decay dominantly with $100 \%$ BR whenever it is kinematically allowed.

(iii) $\tilde{\mathbf{t}_{\mathbf{1}}} \rightarrow \mathbf{b} \ell \tilde{\nu}_{\ell}:$ This decay takes place via off shell $\tilde{W}_{1}$ (decay mediated by off shell $\tilde{W}_{2}$ is suppressed due to its large mass) and dominates when $m_{\tilde{\nu}}+m_{b}<m_{\tilde{t}_{1}}<m_{b}+m_{\tilde{\ell}_{L}}<m_{b}+m_{\tilde{W}_{1}}$ and the decay width is

$$
\Gamma\left(\tilde{t}_{1} \rightarrow b \ell \tilde{\nu}_{\ell}\right)=\frac{\left(\alpha V_{11}\right)^{2}}{16 \pi \sin ^{4} \theta_{W} m_{\tilde{t}_{1}}} \int W\left(x_{b}, x_{\ell}\right) d x_{\ell} d x_{b}
$$

where

$$
\begin{aligned}
W\left(x_{b}, x_{\ell}\right) & =\frac{1}{\left(1+\mu_{b}-x_{b}-\mu_{\tilde{W}_{1}}\right)^{2}} \\
& \times\left[c_{L}^{2} m_{\tilde{W}_{1}}^{2}\left(1+\mu_{\tilde{\nu}}-\mu_{b}-2+x_{b}+x_{\ell}\right)\right. \\
& +2 c_{L} c_{R} m_{\tilde{W}_{1}} m_{b}\left(1+\mu_{b}-\mu_{\tilde{\nu}}-x_{b}\right) \\
& +c_{R}^{2} m_{\tilde{t}_{1}}^{2}\left(1-\mu_{b}-\mu_{\tilde{\nu}}-x_{\ell}\right)\left(1+\mu_{b}-\mu_{\tilde{\nu}}-x_{b}\right) \\
& \left.-c_{R}^{2} m_{\tilde{\nu}}^{2}\left(1+\mu_{\tilde{\nu}}-\mu_{b}-2+x_{b}+x_{\ell}\right)\right]
\end{aligned}
$$

where $\mu$ 's and $x$ 's are defined below. Here we have neglected the masses of the SM leptons.

(iv) $\tilde{\mathbf{t}_{\mathbf{1}}} \rightarrow \mathbf{b} \nu_{\ell} \tilde{\ell_{\mathbf{L}}}:$ This decay occurs through off shell $\tilde{W}_{1}$ and dominates when $m_{\tilde{\ell}_{L}}+$ $m_{b}<m_{\tilde{t}_{1}}<m_{b}+m_{\tilde{W}_{1}}$ and the decay rate is given by

$$
\Gamma\left(\tilde{t}_{1} \rightarrow b \tilde{\ell}_{L} \nu_{\ell}\right)=\frac{\left(\alpha U_{11}\right)^{2}}{16 \pi \sin ^{4} \theta_{W} m_{\tilde{t}_{1}}} \int W\left(x_{b}, x_{\tilde{\ell}}\right) d x_{\tilde{\ell}} d x_{b}
$$


where

$$
\begin{aligned}
W\left(x_{b}, x_{\ell}\right) & =\frac{1}{\left(1+\mu_{b}-x_{b}-\mu_{\tilde{W}_{1}}\right)^{2}} \\
& \times\left[c_{L}^{2} m_{\tilde{t}_{1}}^{2}\left(1+\mu_{b}-\mu_{\tilde{\ell}}-x_{b}\right)\left(1-\mu_{b}-\mu_{\tilde{\ell}}-2+x_{b}+x_{\tilde{\ell}}\right)\right. \\
& -c_{L}^{2} m_{\tilde{\ell}}^{2}\left(1+\mu_{\tilde{\ell}}-\mu_{b}-x_{\tilde{\ell}}\right)+2 c_{L} c_{R} m_{\tilde{W}_{1}} m_{b}\left(1+\mu_{b}-\mu_{\tilde{\ell}}-x_{b}\right) \\
& \left.+c_{R}^{2} m_{\tilde{W}_{1}}^{2}\left(1+\mu_{\tilde{\ell}}-\mu_{b}-x_{\tilde{\ell}}\right)\right]
\end{aligned}
$$

The couplings $c_{L}$ and $c_{R}$ are the same as in Eq. 15 and

$$
\begin{array}{ccc}
\mu_{b}=m_{b}^{2} / m_{\tilde{t}_{1}}^{2} & \mu_{\ell(\tilde{\ell})}=m_{\ell}^{2} / m_{\tilde{t}_{1}}^{2} & \mu_{\tilde{\nu}}=m_{\tilde{\nu}}^{2} / m_{\tilde{t}_{1}}^{2} \\
x_{b}=2 E_{b} / m_{\tilde{t}_{1}} & x_{\ell(\tilde{\ell})}=2 E_{\ell} / m_{\tilde{t}_{1}} & x_{\tilde{\nu}}=2 E_{\tilde{\nu}} / m_{\tilde{t}_{1}} \\
\text { with } & x_{b}+x_{\ell(\tilde{\ell})}+x_{\tilde{\nu}(\nu)}=2 &
\end{array}
$$

The range of integration of the above Eq. 16, 18 are 16

$$
\begin{aligned}
& 2 \sqrt{\mu_{b}} \leq x_{b} \leq 1+\mu_{b}-\mu_{c}-\mu_{d}-2 \sqrt{\mu_{c} \mu_{d}} \\
& \frac{\left(2-x_{b}\right)\left(1+\mu_{b}+\mu_{c}-\mu_{d}-x_{b}\right)-\sqrt{\left(x_{b}^{2}-4 \mu_{b}\right) \lambda\left(1+\mu_{b}-x_{b}, \mu_{c}, \mu_{d}\right)}}{2\left(1-x_{b}+\mu_{b}\right)} \leq x_{c} \\
& \leq \frac{\left(2-x_{b}\right)\left(1+\mu_{b}+\mu_{c}-\mu_{d}-x_{b}\right)+\sqrt{\left(x_{b}^{2}-4 \mu_{b}\right) \lambda\left(1+\mu_{b}-x_{b}, \mu_{c}, \mu_{d}\right)}}{2\left(1-x_{b}+\mu_{b}\right)}
\end{aligned}
$$

where $c=\ell(\tilde{\ell})$ and $d=\tilde{\nu}(\nu)$ for Eq. 16 ( 18).

It is not difficult to understand the behaviour of the above two three-body decay modes of $\tilde{t}_{1}$ (Eq. 16 and Eq. 18 (if both are kinematically allowed)) qualitatively by simply examining the dimension of the interaction Lagrangians for both the processes 8 . It is to be noted that chargino does not interact with the right handed charged sleptons if one neglects the masses of corresponding SM leptons. The interaction Lagrangians for these two decay modes contain terms like $\tilde{t}_{L} \bar{b}_{L} l_{L} \tilde{\nu}_{L}^{*}$ for (iii) and $\tilde{t}_{L} \bar{b}_{L} \bar{\nu}_{l} \tilde{l}_{L}$ for (iv). In the former case one needs a $\gamma$ matrix to contract the chirality between the fermion and the antifermion and that has to be, in turn, contracted with the derivative of the scalar field. Therefore, on dimensional ground, a coefficient $\sim m^{-2}$ is necessary in this term. On the other hand, in the charged slepton case the operator does not require any derivative and it has dimension five, and hence it requires only a coefficient $\sim m^{-1}[8]$. Hence the decay mode $\tilde{t_{1}} \rightarrow b \ell \tilde{\nu}$ (Eq. 16) is always suppressed by an extra mass dimension in comparison to the charged slepton decay mode viz. $\tilde{t_{1}} \rightarrow b \nu \tilde{\ell}$ (Eq. 18). Naturally, whenever the latter mode (Eq. 18) is open it dominates over the former (Eq 16) in this scenario. We have checked this by calculating numerically the decay widths as given by Eqs. 16 and 18 . 
In the MSSM, the masses of charginos and neutralinos and their corresponding mixing matrix elements $U, V$ and $N$ can be determined by $M_{2}, \mu$ and $\tan \beta$. Here $M_{2}$ is the $S U(2)$ gaugino mass and it is related to $M_{1}$, the $U(1)$ gaugino mass, in the following manner at the electroweak scale [17] under the assumption of gaugino mass unification at the GUT scale - -

$$
M_{1}=\frac{5}{3} M_{2} \tan ^{2} \theta_{W}
$$

where $M_{1}$ is the $U(1)$ gaugino mass. There is also experimental lower bound on the mass of the lighter chargino from the non observation of SUSY events at LEP experiments. Interestingly, limit on the chargino mass is also related to $m_{\tilde{\nu}}$. In the case of chargino pair production through $e^{+} e^{-}$collision, in addition to the $s$-channel diagrams, there is also a $t$-channel diagram (via $\tilde{\nu}$ exchange) which interferes destructively with the $s$-channel and hence, reduces the cross section. When $m_{\tilde{\nu}}$ is larger $(\geq 200 \mathrm{GeV})$ than $m_{\tilde{W}_{1}}$ the effect is less pronounced. But, for $m_{\tilde{\nu}}<m_{\tilde{W}_{1}}$ the chargino pair production cross section will reduce and also the leptonic branching ratio of $\tilde{W}_{1}$ (Eq. 国) will get enhanced and finally will reduce the limit of $m_{\tilde{W}_{1}}$. The present available lower limit of lighter chargino obtained by LEP experiments at $\sqrt{s}=160 \mathrm{GeV}$ and $172 \mathrm{GeV}[18,19]$ are

$$
\begin{aligned}
m_{\tilde{W}_{1}}>85 \mathrm{GeV} & \text { for } \\
>67 \mathrm{GeV} & \text { for } 41 \mathrm{GeV}<m_{\tilde{\nu}}<100 \mathrm{GeV}
\end{aligned}
$$

It is to be noted that the bound on lighter chargino in the case of light sneutrino holds when mass difference between them is greater than $10 \mathrm{GeV}$. Therefore, for almost degenerate sneutrino and lighter chargino with the lighter chargino heavier than the sneutrino there is no such bound. The only relevent bound on chargino mass then comes from LEP-1 which is little better than $M_{Z} / 2$. As in our present analysis we consider the case of light sneutrino $\left(m_{\tilde{\nu}}<100 \mathrm{GeV}\right)$, the only relevant limit on $\tilde{W}_{1}$ is the second one. In the stop sector, we have used $m_{\tilde{t}_{1}}$ and $\theta_{\tilde{t}}$ as input parameters. The ALEPH collaboration [13] at LEP has come about with a lower bound on $m_{\tilde{t}_{1}}$

$$
m_{\tilde{t}_{1}}>70 \mathrm{GeV}
$$

analysing the data taken upto $\sqrt{s}=172 \mathrm{GeV}$ in the channel $\tilde{t}_{1} \rightarrow b \ell \tilde{\nu}$ assuming a mass difference between the $\tilde{t}_{1}$ and $\tilde{\nu}$ of at least $10 \mathrm{GeV}$. This bound is independent of the mixing angle $\theta_{\tilde{t}}$ in the stop sector. On the other hand, DØ collaboration at Fermilab have also constrained $m_{\tilde{t}_{1}}-m_{\tilde{Z}_{1}}$ plane analysing their data for $\tilde{t}_{1} \rightarrow c \tilde{Z}_{1}$ in jets $+\not_{T}$ channel [20]. As described above, those $\mathrm{D} \varnothing$ constraints on $m_{\tilde{t}_{1}}$ will not work in our analysis. They have also carried out search for stop squark [21] in the dielectron channel assuming the conventional cascade decay of stop(see, Eq. ??). But effectivley, no limit on $m_{\tilde{t}_{1}}$ is set. The other very crucial parameter of our analysis is $m_{\tilde{\nu}_{\ell}}$. The light sneutrino scenario can 
be easily accommodated if one embeds GUT in the MSSM. Further, with the assumption of a common scalar mass $\left(m_{0}\right)$ at the GUT scale the slepton masses get related 22 at the electroweak scale and the slepton masses at the electroweak scale as -

$$
\begin{aligned}
& m_{\tilde{\ell}_{R}}^{2}=m_{0}^{2}+0.22 M_{2}^{2}-\sin ^{2} \theta_{W} M_{Z}^{2} \cos 2 \beta \\
& m_{\tilde{\ell}_{L}}^{2}=m_{0}^{2}+0.75 M_{2}^{2}-0.5\left(1-2 \sin ^{2} \theta_{W}\right) M_{Z}^{2} \cos 2 \beta \\
& m_{\tilde{\nu}_{\ell}}^{2}=m_{0}^{2}+0.75 M_{2}^{2}+0.5 M_{Z}^{2} \cos 2 \beta
\end{aligned}
$$

where $\ell$ is $e$ or $\mu$. In case of staus, the mixing plays an important role for very large $\tan \beta$ and $\mu$ parameter. In some region of the $m_{0}, M_{2}$ and $\tan \beta$ parameter space the masses of charged sleptons $\left(\tilde{\ell}_{L}, \tilde{\ell}_{R}\right)$ and also that of $\tilde{\nu}$ may be lighter than all the gauginos except the LSP. It happens due to the mass-splittings in the presence of $S U(2)$ breaking $D$-terms. It is to be noted that the above relations hold in a model independent way as long as $S U(2)_{L}$ is a good symmetry at the electroweak scale. The light slepton masses are much more natural if one allows non-universal soft breaking masses 23] and in that case our light slepton scenario holds in a larger region of the allowed parameter space.

The ALEPH collaboration[24] has carried out direct searches for right handed $\operatorname{selectron}\left(\tilde{e}_{R}\right)$ and smuon $\left(\tilde{\mu}_{R}\right)$ in the opposite sign dilepton plus missing energy channel at energies $\sqrt{s}=$ $161 \mathrm{GeV}$ and $172 \mathrm{GeV}$. From the non-observation of events they have obtained lower limits on $m_{\tilde{e}_{R}}$ and $m_{\tilde{\mu}_{R}}$. The absolute values of the limits depend on the relative mass differences between the sleptons and the LSP. Since the slepton masses are determined by two parameters $M_{2}$ and $m_{0}$ for a given $\tan \beta$, the limits on slepton masses can be translated so as to constrain the $m_{0}-M_{2}$ plane. In Ref. [24] the excluded region in the $m_{0}-M_{2}$ plane has been shown for $\mu=-200 \mathrm{GeV}$ and $\tan \beta=2$. In our analysis we have used this constrained $m_{0}-M_{2}$ parameter space to determine $m_{\tilde{\nu}_{\ell}}$ and $m_{\tilde{\ell}_{L}}$ which are subsequently used as inputs to our analysis.

\section{Results and Discussion}

At Tevatron the dominant mechanisms for stop production are the leading order QCD processes like quark-antiquark annihilation and gluon-gluon fusion 25.

$$
q \bar{q} \rightarrow \tilde{t}_{1} \tilde{t}_{1} ; g g \rightarrow \tilde{t}_{1} \tilde{t}_{1}
$$

The next to leading order(NLO) corrections to these processes has been computed recently 26]. This correction is only a few percent and is positive. In our conservative estimate we have not taken into account this NLO correction. The cross section for stop pair production depends only on $m_{\tilde{t}_{1}}$ and its dependence on other MSSM parameters is almost negligible even 
it does not depend on $\theta_{\tilde{t}}$ as it is mainly a QCD process. So, any bound obtained on the stop production cross section from experiment can be translated to a bound on the stop mass in a straight forward manner. In our calculation we have used the CTEQ3L[27] for parton density setting the QCD scale at $m_{\tilde{t}_{1}}$.

Once a pair of stop squarks is produced at the Tevatron, depending on its mass, the stop squark will decay through either of the channels (ii)-(iv) as described in section 1, essentially with $100 \%$ branching ratio in the light slepton scenario. If $m_{\tilde{t}_{1}}<m_{\tilde{W}_{1}}+m_{b}$, then $\tilde{t}_{1}$ will decay either via the channel in Eq. 17 or in Eq. 8 depending on $m_{\tilde{\nu}}$ and $m_{\tilde{\ell}}$, as discussed in the last section. If $m_{\tilde{t}_{1}}>m_{b}+m_{\tilde{W}_{1}}$, then $\tilde{t}_{1}$ will decay through channel $\tilde{t_{1}} \rightarrow b \widetilde{W_{1}}$ (Eq. 1) and subsequently $\tilde{W}_{1}$ will decay as indicated in Eq. 团or Eq. . . As a consequence, pair production of $\tilde{t}_{1}$ eventually leads to a signal consisting of opposite sign dilepton $\left(\ell^{+} \ell^{-}\right)$and jets(mainly $b$-jets) along with missing transverse momentum $\left(p_{T}\right)$ due to the presence of $\tilde{\nu}$ and/or $\tilde{Z}_{1}$. The dilepton event from $t \bar{t}$ pair production is topologically similar to this and hence acts as the dominant SM background to this signal. The other sources of the SM backgrounds are $W$-pair production and the Drell-Yan processes. We have mentioned in the introduction that the signal, consisting same final states for stop squarks at the Tevatron, although in a different scenario, has been studied in Ref. [9] where cascade decays of $\tilde{W}_{1}$ are considered as in Eq. 3. In that analysis the authors optimised a set of kinematic cuts by which the SM backgrounds can be minimised without much affecting the signal rates. In our parton level analysis we have used those optimised cuts[9], viz.

1. $p_{T}^{\ell}>10 \mathrm{GeV},\left|\eta_{\ell}\right|<1$ and $E_{A C}^{T}<10 \% p_{T}^{\ell}$

2. $20^{\circ}<\phi_{\ell^{+} \ell^{-}}<160^{\circ}$

3. $\not p_{T}>25 \mathrm{GeV}$

4. $B<100 \mathrm{GeV}$ where $B=p_{T}^{\ell_{1}}+p_{T}^{\ell_{2}}+\not p_{T}$

5. number of jets, $n_{j} \geq 1$ with $p_{T}^{j}>15 \mathrm{GeV}$ and $\left|\eta_{j}\right|<2$

The lepton selection cut (1) has been applied to both the leptons. The cut (2) removes the unlike sign dileptons due to the Drell-Yan process where lepton pairs emerge back to back most of the time. We have already mentioned that the most significant background comes from $t \bar{t}$ and $W W$ productions where the leptons come from real $W$ 's. For the signal process, the leptons come either from the 3-body decay of $\tilde{t}_{1}$ (see Eqs.7-8) or from the 2 body decay of $\tilde{W}_{1}$ (see Eqs.4-6). Hence the leptons are always accompanied by a massive particle which is either a $\tilde{\nu}$ or a $\tilde{Z}_{1}$. Therefore, it is expected that the leptons in the signal are softer than that from the backgrounds. Therefore, by imposing cut on a newly constructed kinematic 
variable like " $B$ ", which is the scalar sum of the transverse momenta of two leptons and $p_{T}$, one can cope with the backgrounds efficiently. Obviously, in the case of signal, leptons are distributed towards the lower values of " $B$ " whereas in the case of background the situation is just the opposite. Hence, putting an upper cut(4) on " $B$ " can effectively reduce the background without killing the signal significantly 9]. Cut (5), which is the requirement of at least one jet with $p_{T}$ greater than $15 \mathrm{GeV}$, effectively suppresses the Drell-Yan background. The estimated cross sections for the SM backgrounds from $t \bar{t}$ and $W W$ production under all these cuts as described above are $14 \mathrm{fb}$ for $m_{t}=170 \mathrm{GeV}$ and $10 \mathrm{fb}$ respectively [9] and we have used these numbers in our analysis. Note that the value of $m_{t}$ conforms with recent experiments 28.

As for an illustration, we have computed the signal cross section at $\sqrt{s}=1.8 \mathrm{TeV}$ for a set of input parameters given in Table 1. In our analysis we have fixed $\theta_{\tilde{t}}=-45^{\circ}, \mu=-200 \mathrm{GeV}$. The signal rate is not too sensitive to $\theta_{\tilde{t}}$ since it neither affects the cross section nor the BR of $\tilde{t}_{1}$ decay which is always nearly $100 \%$ for a particular decay channel. This is so because out of various allowed decay channels for $\tilde{t}_{1}$ only one dominates overwhelmingly over the others at a time. We have estimated the signal cross section for different $m_{\tilde{t}_{1}}$ ranging from 80-170 GeV keeping in mind its lower bound from LEP(Eq. 27). If $m_{\tilde{t}_{1}}>m_{t}$ then the decay mode (viii), as described in section 1, will open up and consequently the signal will be different. The hardness of the lepton momentum depends on the relative mass differences $\Delta m_{\tilde{\nu}}=m_{\tilde{W}_{1}}-m_{\tilde{\nu}}\left(\right.$ for $\tilde{t}_{1}$ decay via on shell $\tilde{W}_{1}$ ) or $\Delta m_{\tilde{\ell}}=m_{\tilde{\ell}}-m_{\tilde{Z}_{1}}$. The larger is the value of this mass difference, harder are the leptons. In our analysis we considered $e e, \mu \mu$ and $e \mu$ dilepton final states i.e. $40 \%$ of stop pair production events will have final states with dileptons.

In Fig. 1 we have shown the $p_{T}$ distribution of the lepton for some representative values of $\left(m_{\tilde{t}_{1}}, m_{\tilde{\nu}}, m_{\tilde{W}_{1}}\right)=\mathrm{A}(105,69,90) \mathrm{GeV} ; \mathrm{B}(105,81.4,90) \mathrm{GeV} ; \mathrm{C}(130,69,90) \mathrm{GeV} ; \mathrm{D}(130,81.4,90)$ $\mathrm{GeV}$. The distributions obtained are subject to the kinematic cuts as described above except for the $p_{T}$ cuts on the lepton pairs. For a fixed $m_{\tilde{t}_{1}}$, the $p_{T}$ of lepton in case of point $\mathrm{A}$ is harder than in case of point $\mathrm{B}$ since $\Delta m_{\tilde{\nu}}$ is larger for the former. Similar behaviour exists for the points $\mathrm{C}$ and $\mathrm{D}$. As far as the distributions corresponding to $\mathrm{A}$ and $\mathrm{C}$ are concerned, although $\Delta m_{\tilde{\nu}}$ 's are the same, the distribution $\mathrm{C}$ takes over the distribution $\mathrm{A}$ in the higher $p_{T}$ region. This enhancement is due to large $m_{\tilde{t}_{1}}$ which has boosted the lepton in case C. But this behaviour does not show up in cases B and D where the extra boost due to large $m_{\tilde{t}_{1}}$ for the point $\mathrm{D}$ does not help as the lepton are very much less energetic.

It is obvious from Fig. 1 that cut (1) on lepton will have significant bearings over case B and $\mathrm{D}$ and is less significant for $\mathrm{A}$ and $\mathrm{C}$.

In Fig.2 the signal cross sections for different $m_{\tilde{t}_{1}}$ values are presented for the first four 
sets of parameters of Table 1. For each of the curves the signal cross section goes up with increasing $m_{\tilde{t}_{1}}$ as long as $\tilde{t}_{1}$ is light despite a decrease in stop pair production cross section. This is because the cut efficiency factor goes up for larger $m_{\tilde{t}_{1}}$ as the leptons and jets become harder for a given set of input parameters. What actually happens in such a region is that the reduction of cross section is overcompensated for by the enhancement in the efficiency of the kinematic cuts. As for example, for the set (a) the stop pair production cross section decreases by a factor of 2 as $m_{\tilde{t}_{1}}$ goes from 80 to $90 \mathrm{GeV}$, whereas the cut efficiency factor increases by an even larger factor. It happens since for larger $m_{\tilde{t}_{1}}$ harder are the $b$-jets and the leptons. The 'dip's occur in cases (a) and (d) just where on shell $\tilde{W}_{1}$ 's are produced from stop decay for some values of $m_{\tilde{t}_{1}}$ (see Eq. 1), while small $m_{\tilde{t}_{1}}-m_{\tilde{W}_{1}}$ leads to very soft $b$-jets and the signal gets severely affected by the jet selection cut. However, such 'dip' also appears in the curves for the sets (b) and (c) but with negligibly small cross sections and we have not shown it. Note that the the signal cross section dominates over the background (24 fb) for stop masses upto about $135 \mathrm{GeV}$ for a less favourable region of the pa space and nearly $150 \mathrm{GeV}$ for the most favourable one having few events for the present integrated luminosity of $110 \mathrm{pb}^{-1}$. Also, it is not possible to probe stop mass in the region of parameter space where the 'dip' occurs. It is to be noted that in the conventional scenario stop mass up to $100 \mathrm{GeV}$ can be probed with the same luminosity option 9] with few events. In our scheme, the signal will be viable when $m_{\tilde{t}_{1}}-m_{\tilde{\nu}}$ (for off shell $\tilde{W}_{1}$ ) or $m_{\tilde{t}_{1}}-m_{\tilde{W}_{1}}$ (for on shell $\left.\tilde{W}_{1}\right)$ is larger than at least $10 \mathrm{GeV}$. Notice that for small $m_{\tilde{t}_{1}}(\leq 100 \mathrm{GeV})$ with $m_{\tilde{t}_{1}}>>m_{\tilde{\nu}}$, if this scenario is kinematically allowed, it will give a few 100 events(see the case for (a) in Fig.2) for the present luminosity . We want to emphasise that if this light slepton scenario is at all instrumental for the cascade decays of stop squark then it may turn out as one of its viable discovery channels at Tevatron although over a rather limited region of the MSSM parameter space. The signal size has also a modest dependence on the MSSM parameters through $m_{\tilde{\nu}}, m_{\tilde{W}_{1}}$ and $m_{\tilde{Z}_{1}}$. But $m_{\tilde{W}_{1}}$ does not vary so much with $\mu$ in the gauge dominated region i.e. when $M_{2} \gg|\mu|$. Hence, signal size is not that sensitive to $\mu$ parameter. However, in the negative $\mu$ region $m_{\tilde{W}_{1}}$ decreases with the increase of $\tan \beta$.

In Fig. 3 we have shown the signal cross section with the conventions similar to Fig.2 but for $\tan \beta=10$. We have computed the signal rate for the set of points (e) and (f) of Table 1. Although for higher $\tan \beta$ the constrained region in the $m_{0}-M_{2}$ plane is not available from slepton search at LEP [24], still one can qualitatively argue that the value which we have used is very much likely to be allowed by the present lower limits on the mass of the right handed sleptons. This is because the increase of $\tan \beta$ for a given $m_{0}$ and $M_{2}$ lowers the value of $m_{\tilde{\nu}}$ whereas it enhances $m_{\tilde{e}_{L}}$ and $m_{\tilde{\mu}_{R}}$. The signal rate for set (e) decreases in comparison to set (a) since in the former case $\Delta m_{\tilde{\nu}}$ is smaller. On the other hand, for higher 
$\tan \beta$, the signal in case (f) is stronger than that in set (c). In case (c), leptons may also come from the charged slepton decays (Eqs. 5-6), which involve further cascading compared to case (f) and hence may become soft. For this higher $\tan \beta$ case the signal rate is above the background level for $m_{\tilde{t}_{1}}$ upto about $130 \mathrm{GeV}$.

In the Main Injector (MI) upgrade the integrated luminosity is expected to be enhanced by a factor of 20 which will result in more number of events. But, unfortunately, the discovery range of $m_{\tilde{t}_{1}}$ is not expected to extend as the signal in this channel is highly limitted by the backgrounds. The message from this work is that one can constrain the relative differences among $m_{\tilde{t_{1}}}, m_{\tilde{\nu}}$ and $m_{\tilde{W}_{1}}$ for light $\tilde{t_{1}}$ simply by analyzing the present dilepton data from Tevatron in the light slepton scenario. Especially, for lower values of $m_{\tilde{t}_{1}}$, the difference $m_{\tilde{t}_{1}}-m_{\tilde{\nu}}$ can be constrained analysing the same set of data whence one can put a lower bound on $m_{\tilde{\nu}}$ if the limit of $m_{\tilde{t}_{1}}$ can be found from any other experiments, say, from the LEP or vice versa.

In conclusion, we have investigated the decay patterns of the light stop when the sleptons are lighter than it and the charginos as well. We have studied the prospect of stop search at Tevatron in such a scenario although the latter is viable only for an additionally constrained, but still significant, region of the MSSM parameter space. We observe that light stop masses upto $140 \mathrm{GeV}$ can be probed in some favourable region of this parameter space with few events. Moreover, it is possible to constrain the light slepton scenario in the minimal supergravity (mSUGRA) inspired MSSM from the non-observation of any signal in this channel in the present data set at Tevatron.

\section{Acknowledgements}

The authors are indebted to Amitava Datta for many valuable suggestions and careful reading of the manuscript. AD acknowledges financial assistance from the Department of Science and Technology, Government of India. MG was supported in part by a 1997 foreign posdoctoral fellowship through the Korean Science and Engineering Foundation, and in part by a graduate school fellowship from Yonsei University, Seoul. 


\section{References}

[1] For reviews see e.g. H. E. Haber and G. L. Kane, Phys. Rep. 117, 75(1985); H. P. Nilles, Phys. Rep. 111, 1 (1984).

[2] See, for example, A. Altarelli, R. Barbieri, and F. Caravaglios, Int. J. Mod. Phys. A13, 1031, 1998 (and references there in).

[3] J. F. Gunion and H. E. Haber, Nucl. Phys. B272, 1(1986).

[4] S. Weinberg, Phys. Rev. D26, 287 (1982); N. Sakai and T. Yanagida, Nucl. Phys. B197, 83 (1982); S. Dimopoulos, S. Raby and F. Wilczek, Phys. Lett. B212, 133 (1982).

[5] C. Caso et. al, Eur. Phys. J. C3 1 (1998).

[6] CDF Collaboration, F. Abe et al., Phys. Rev. D56, 1357 (1997); DØ Collaboration, S. Abachi. et al., Phys. Rev. Lett. 75, 618 (1995).

[7] J. Ellis and S. Rudaz, Phys. Lett. 128B, 248 (1983); G. Altarelli and R. Ruckl, Phys. Lett. 144B, 126 (1984).

[8] K. Hikasa and M. Kobayashi, Phys. Rev. D36, 724 (1987).

[9] H. Baer, J. Sender and X. Tata, Phys. Rev. D50, 4517 (1994).

[10] H. Baer, M. Drees, J. Gunion, R. Godbole and X. Tata, Phys. Rev. D44, 725 (1991).

[11] G. Mahlon and G. L. Kane, Phys. Rev. D55, 2779 (1997); S. Mrenna and C. P. Yuan, Phys. Lett. B367, 188 (1996).

[12] See, e.g. L. E. Ibanez and G. G. Ross, Phys. Lett. B110, 215 (1982); L. E. Ibanez and J. Lopez, Nucl. Phys. B233, 511 (1984); M. Drees and M. M. Nojiri, Nucl. Phys. B369, 54 (1992); N. Polonosky and A. Pomarol, Phys. Rev. D51, 6532 (1995); Y. Kawamura, H. Murayama and M. Yamaguchi, Phys. Rev. D51, 1337 (1995)

[13] ALEPH Collaboration, R. Barate et al., Phys. Lett. B413, 431 (1997).

[14] A. Datta, B. Mukhopadhyaya and M. Guchait, Mod. Phys. Lett., 10, 1011 (1995); A. Datta, M. Drees and M. Guchait, Z. Phys. C69, 347 (1996); S. Chakrabarty, A. Datta and M. Guchait, Z. Phys. C68, 325 (1995); A. Datta, Aseshkrishna Datta, S. Raychaudhuri, Phys. Lett. B349, 113 (1995); A. Datta, Aseshkrishna Datta, S. Raychaudhuri, Eur. Phys. J., C1, 375 (1998). 
[15] See, Haber and Kane in Ref.[1].

[16] V. D. Barger and R. J. N. Phillips, Collider Physics, Addison-Wesley, 1987.

[17] For a micro review see Supersymmetry by H. Haber, in Review of Particle Properties, M. Barnett et al. Phys. Rev. D54, 1 (1996).

[18] ALEPH Collaboration, R. Barate et al., CERN PPE/97-128.

[19] DELPHI Collaboration, P. Abreu et al., Eur. Phys. J. C1, 1, 1998.

[20] DØ Collaboration, S. Abachi et al., Phys. Rev. Lett. 76, 2222 (1996).

[21] DØ Collaboration, S. Abachi et. al., Phys. Rev. D57, 589, 1998.

[22] See M. Drees and M. M. Nojiri in Ref.[12].

[23] A. Datta, M. Guchait and N. Parua, Phys. Lett. B395, 54 (1997); A. Datta, Aseshkrishna Datta and M. K. Parida, Phys. Lett. B431, 347 (1998).

[24] ALEPH Collaboration, R. Barate et al., Phys. Lett. B407, 377, 1997.

[25] G. L. Kane and J. P. Leveille, Phys. Lett. B112, 227 (1982); P. R. Harrison and C. H. Llewellyn-Smith, Nucl. Phys. B213, 223 (1983) [Err: Nucl. Phys. B223, 542 (1983)]; S. Dawson and E. Eichten and C. Quigg, Phys. Rev. D31, 1581 (1985); E. Reya and D. P. Roy, Phys. Rev. D32, 645 (1985).

[26] W. Beenakker, M. Krammer, T. Plehn, M. Spira and P. M. Zerwas, Nucl. Phys. B515, 3 (1998).

[27] H. Plothow-Besch, PDFLIB, Comp. Phys. Comm. 75 396(1993).

[28] CDF Collaboration, F. Abe et al., Phys. Rev. Lett. 79, 1992(1997); F. Abe et al., Phys. Rev. Lett. 80, 2767 (1998). 


\section{Figure Captions}

Fig.1 : The $p_{T}$ distributions for one of the leptons are shown for the values of $\left(m_{\tilde{t}_{1}}, m_{\tilde{\nu}}, m_{\tilde{W}_{1}}\right)$ $=\mathrm{A}(105,69,90) \mathrm{GeV} ; \mathrm{B}(105,81.4,90) \mathrm{GeV} ; \mathrm{C}(130,69,90) \mathrm{GeV} ; \mathrm{D}(130,81.4,90) \mathrm{GeV}$. In case of $\mathrm{A}$ and $\mathrm{C},\left(m_{0}, M_{2}\right)=(55,75) \mathrm{GeV}$ whereas for $\mathrm{B}$ and $\mathrm{D}$ is $\left(m_{0}, M_{2}\right)=(70,75) \mathrm{GeV}$. For all the cases $\tan \beta=2, \mu=-200 \mathrm{GeV}$ and $\theta_{\tilde{t}}=-45^{\circ}$.

Fig.2 : The variations of dilepton signal cross sections are shown for different stop masses at the Tevatron for $\sqrt{s}=1.8 \mathrm{TeV}$. The different labels a,b,c,d correspond to different sets of parameters as described in Table 1 . The values of $\tan \beta$ and $\theta_{\tilde{t}}$ are the same as in Fig.1.

Fig.3 : Same as in Fig.2 except for the parameter sets which are (e) and (f) of Table 1.

Table 1

\begin{tabular}{|c|c|c|c|c|c|c|c|}
\hline Set & $m_{0}$ & $m_{2}$ & $\tan \beta$ & $m_{\tilde{\nu}}$ & $m_{\tilde{\ell}_{L}}$ & $m_{\tilde{W}_{1}}$ & $m_{\tilde{Z}_{1}}$ \\
\hline \hline (a) & 55 & 75 & 2 & 69 & 92.6 & 90 & 41.79 \\
\hline (b) & 70 & 75 & 2 & 81.4 & 102.28 & 90 & 41.79 \\
\hline (c) & 40 & 100 & 2 & 86.6 & 102.19 & 112.38 & 54.56 \\
\hline (d) & 67 & 50 & 2 & 60 & 86.28 & 68 & 28.56 \\
\hline (e) & 55 & 75 & 10 & 56.35 & 97.16 & 70 & 38 \\
\hline (f) & 40 & 100 & 10 & 71 & 106.28 & 90.92 & 50 \\
\hline
\end{tabular}

Table 1: Masses( in GeV) of sneutrino( $(\tilde{\nu})$, left handed charged $\operatorname{slepton}\left(m_{\tilde{\ell}}\right)$, lighter chargino $\left(m_{\tilde{W}_{1}}\right)$ and LSP $\left(m_{\tilde{Z}_{1}}\right)$ are shown for different values of $m_{0}, M_{2}$ and $\tan \beta$. The masses of sleptons have been computed using Eq. 29. The extreme left column labels different sets of parameters alphabatically. 


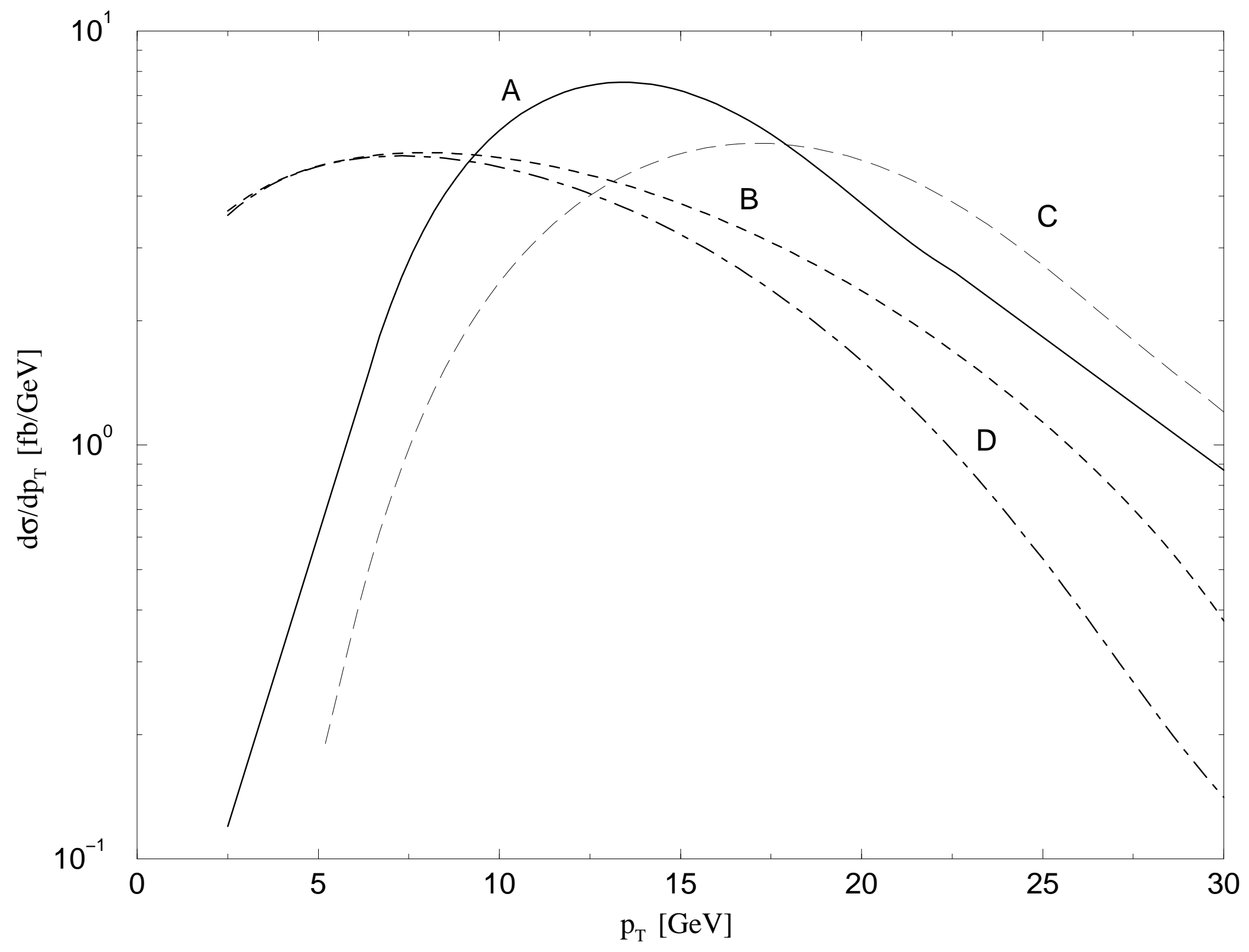

Fig.1 


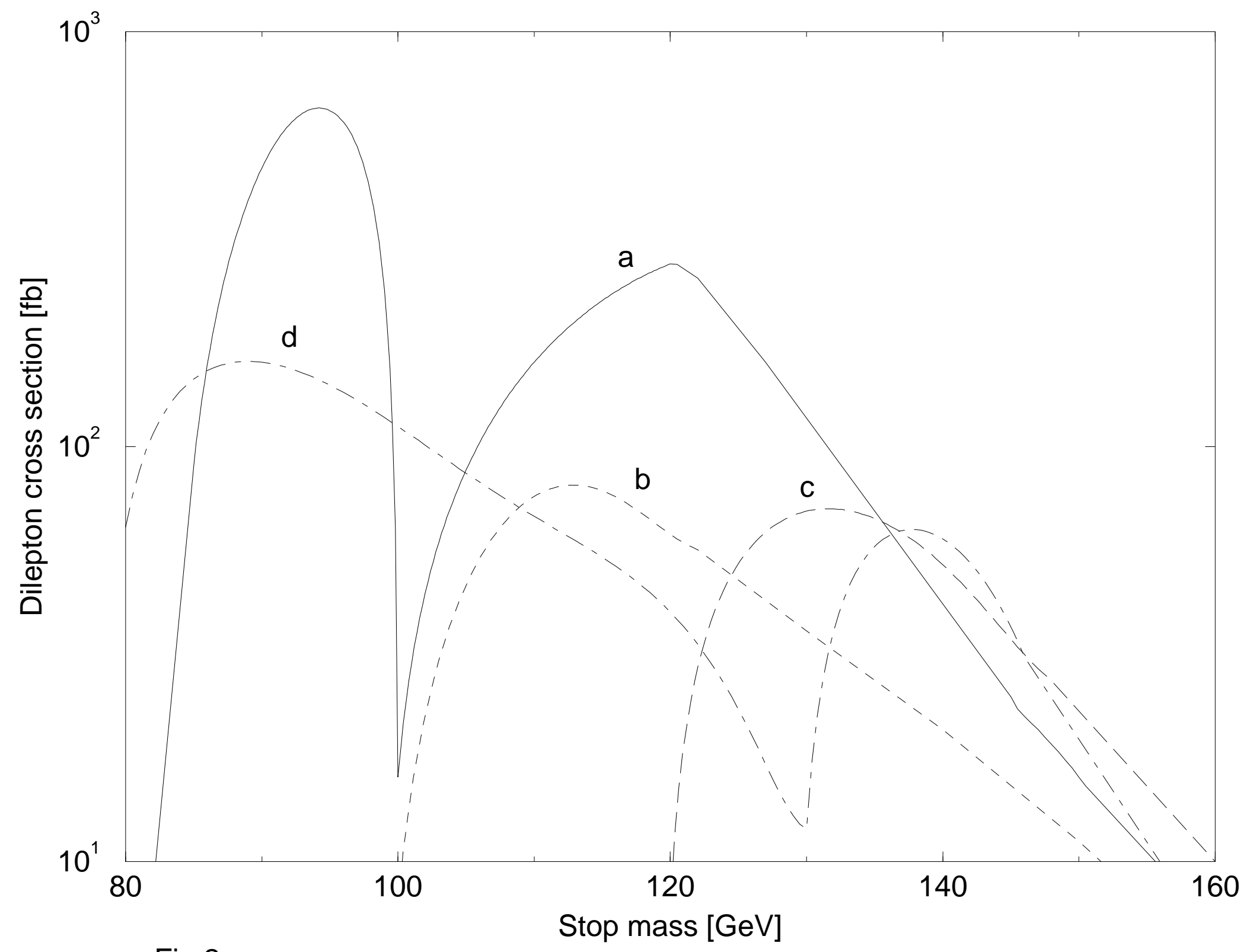

Fig.2 


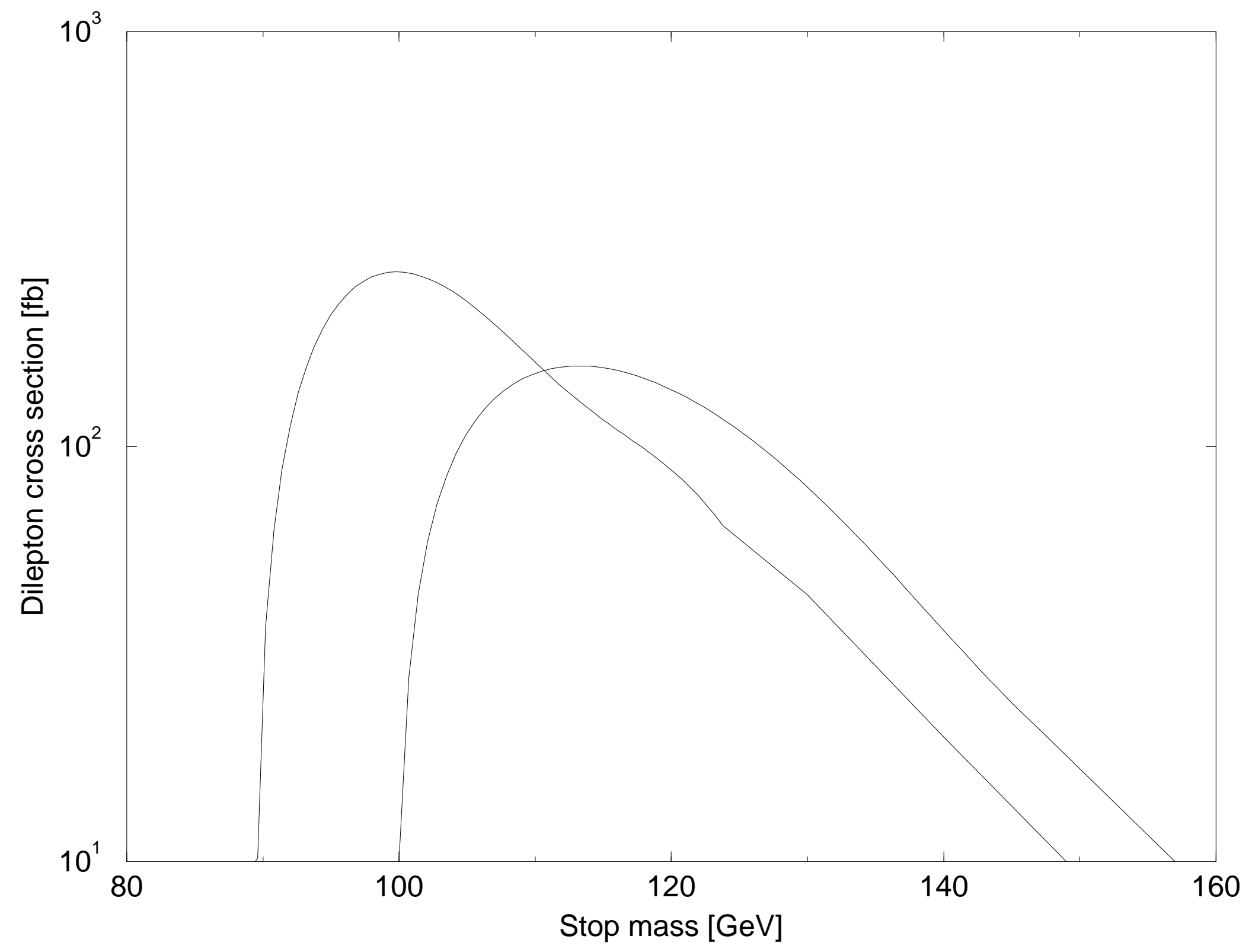

Fig.3 